\title{
Correction to: A pattern learning-based method for temporal expression extraction and normalization from multi-lingual heterogeneous clinical texts
}

Tianyong Hao ${ }^{1,2}$, Xiaoyi Pan ${ }^{1}$, Zhiying $\mathrm{Gu}^{1}$, Yingying $\mathrm{Qu}^{3^{*}}$ and Heng Weng ${ }^{4^{*}}$

\section{Erratum}

After publication of the original article [1] it was noted that the captions relating to Figs. 2 and 3 had been interchanged.

These errors were introduced during typesetting; thus the publisher apologizes for this error. The correct figures and corresponding captions are shown below.

\begin{abstract}
Author details
'School of Information Science and Technology, Guangdong University of Foreign Studies, Guangzhou, China. ${ }^{2}$ School of Computer, South China Normal University, Guangzhou, China. ${ }^{3}$ School of Business, Guangdong University of Foreign Studies, Guangzhou, China. ${ }^{4}$ The Second Affiliated Hospital, Guangzhou University of Chinese Medicine, Guangzhou, China.
\end{abstract}

Published online: 13 April 2018

\section{Reference}

1. Hao T, Pan X, Yi BK, Gu Z, Qu Y, Weng H. A pattern learning-based method for temporal expression extraction and normalization from multi-lingual heterogeneous clinical texts. BMC Med Inform Decis Mak. 2018;18(Suppl 1):22.

* Correspondence: jessie.qu@gdufs.edu.cn; ww128@qq.com

${ }^{3}$ School of Business, Guangdong University of Foreign Studies, Guangzhou, China

${ }^{4}$ The Second Affiliated Hospital, Guangzhou University of Chinese Medicine, Guangzhou, China

Full list of author information is available at the end of the article 

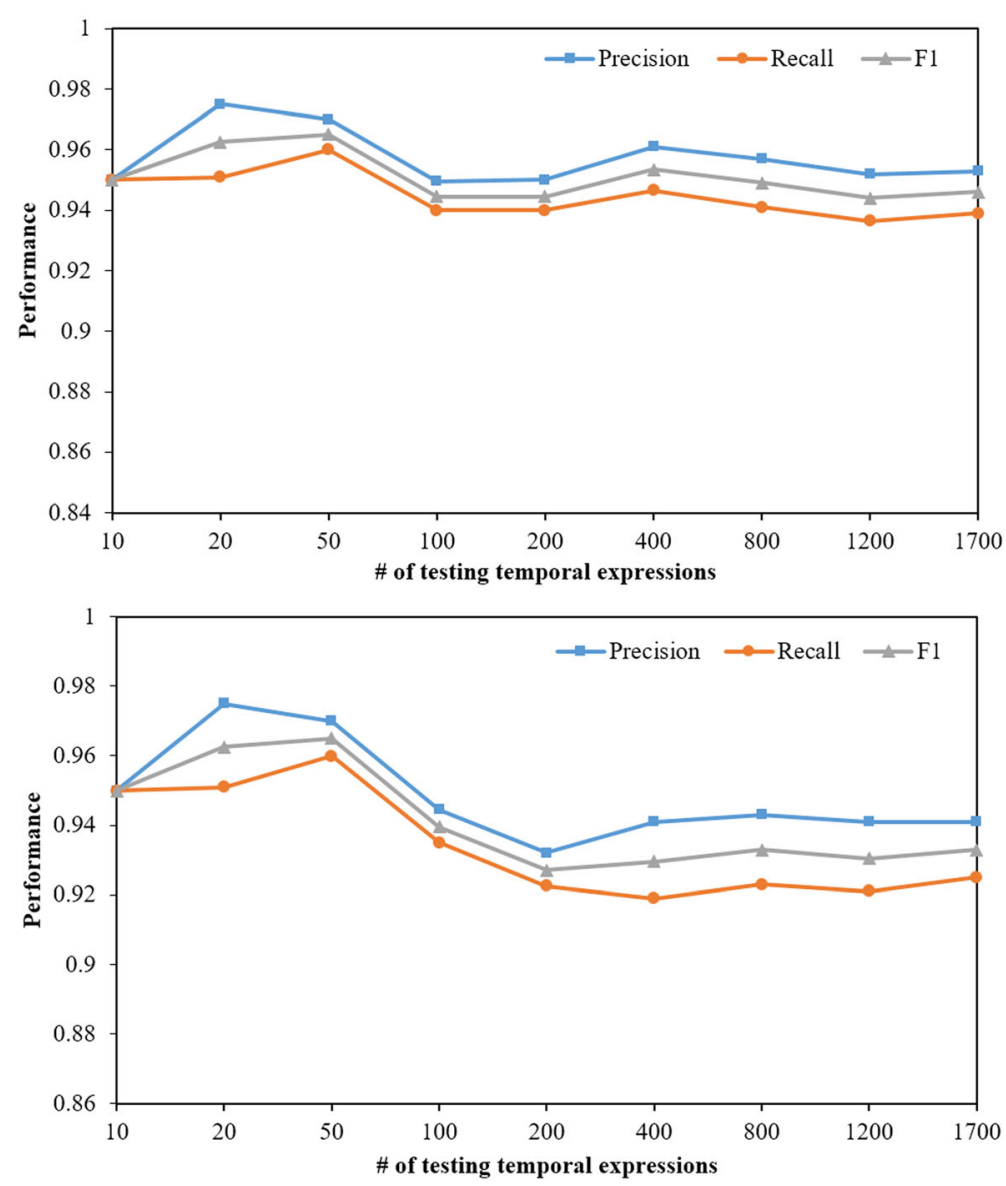

Fig. 2 An implemented graphical user interface of TEER for temporal expression extraction 


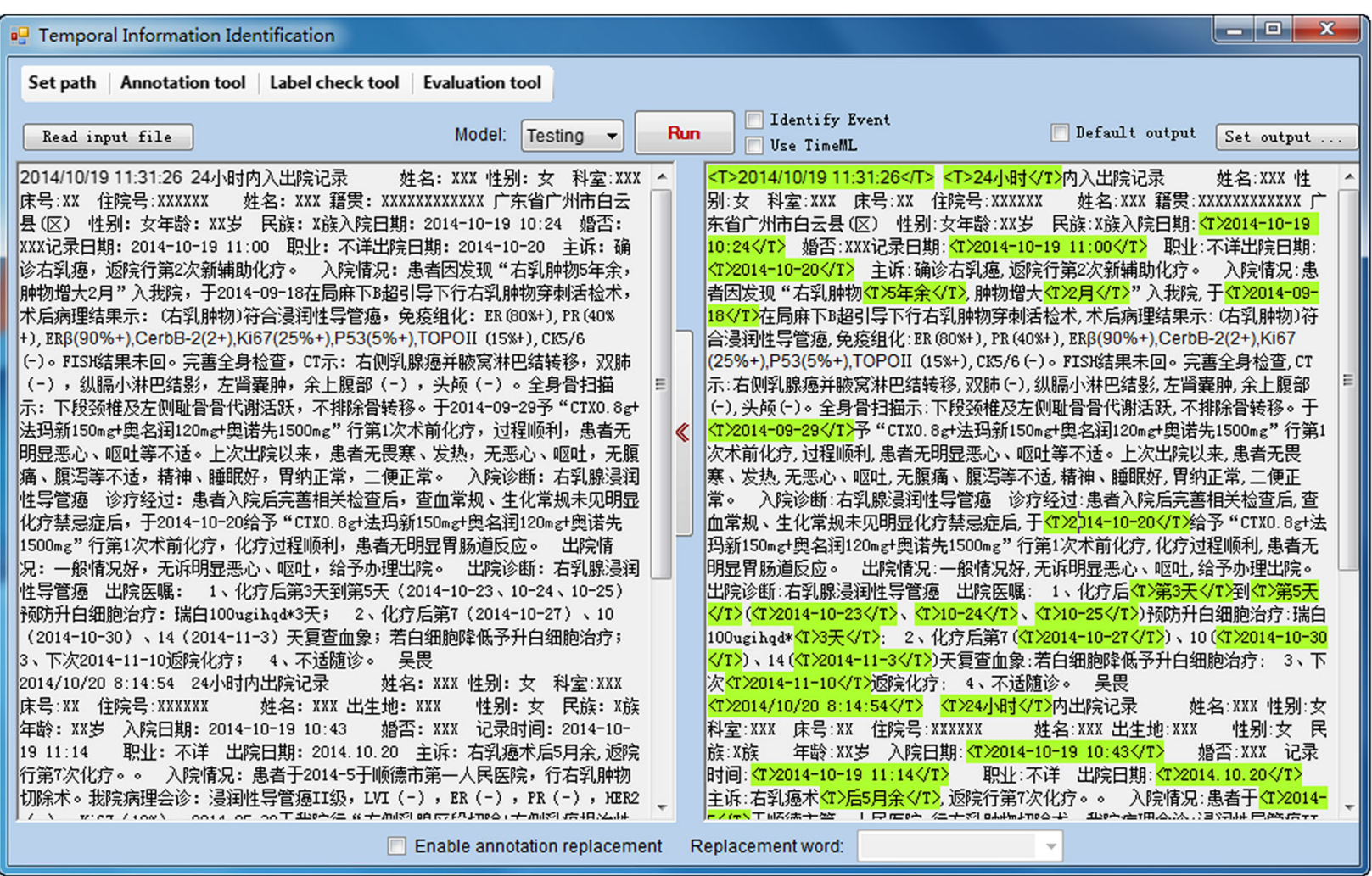

Fig. 3 The scalability evaluation of TEER (the first) and TEER_C (the second) using the increasing number of temporal expressions randomly selected from the testing datasets 